BEATA WACŁAWIK

(Archiwum Państwowe w Olsztynie)

\title{
DAS ARCHIV DER ALBERTUS-UNIVERSITÄT KÖNIGSBERG IM BESTAND DES STAATSARCHIVS IN OLSZTYN (ALLENSTEIN)
}

Nicht als schmückende Krönung des Gebäudes, sondern als wesentliche Bürgschaft für die Zukunft der Staatsschöpfung, die er in Not und Bedrängnis aller Art durch die Verwandelung des Ordenslandes in ein weltliches Herzogtum vollzogen hatte, errichtete Albrecht von Brandenburg 1544 die Universität zu Königsberg ${ }^{1}$.

Mit diesen Worten hat Hans Prutz, Professor für Geschichte und Autor einer Monographie über die Universität im 19. Jahrhundert, die Notwendigkeit der Gründung einer Hochschule in Königsberg erläutert. In der Tat hat Albrecht von Hohenzollern es nicht für Ruhm und Ehre getan, obwohl er als Mäzen der Gelehrten und Künstler sehr bekannt war. Der Herzog hatte andere wichtige

${ }^{1}$ H. Prutz, Die Königliche Albertus-Universität zu Königsberg i. Pr. im neunzehnten Jahrhundert, (1894), S. 1. 
Gründe, die ihn dazu motivierten, neue Lehrzentren zu errichten. Das Herzogtum brauchte Beamte, Lehrer, Theologen, die den neuen Staat formen und die neue religiöse und soziale Identität der Untertanen des Herzogs bilden sollten.

Als Grundlage für die Organisation der Hochschule wurde eine Satzung angenommen, die im Großteil durch Philipp Melanchthon erarbeitet worden war. In dieser Satzung hat der Autor an erster Stelle Theologie als führende Wissenschaft genannt, andere Wissenschaften sollten nach der lutherischen Doktrin unterrichtet werden ${ }^{2}$. Trotz der Bemühungen des Rektors hat die protestantische Hochschule kein Stiftungsprivilegium bekommen, weder vom Papst noch vom Kaiser. Erst der polnische König Zygmunt August hat ihr am 28. März 1560 einen Rechtsstatus gewährt, nach dem Vorbild des Status der Krakauer Universität ${ }^{3}$. Die ersten Magistertitel, die seit 1548 verliehen wurden, waren also rechtswidrig ${ }^{4}$.

Die Studenten in Königsberg hatten vier Fakultäten zur Wahl: Theologie, Medizin und Jura, die sogenannten oberen Fakultäten und eine untere Fakultät - Philosophie, die als studium generale gegründet wurde. In den ersten Jahren haben sich an der Hochschule 20 bis 40 Kandidaten in einem Semester gemeldet, und am Ende des nächsten Jahrhunderts wurden durchschnittlich 50 bis 60 Studenten im Semester angenommen ${ }^{5}$. Das 17. Jahrhundert

${ }^{2}$ W. Hubatsch, Die Albertus-Universität zu Königsberg i. Pr. in der deutschen Geistesgeschichte 1544-1944, in: Deutsche Universitäten und Hochschulen im Osten, hg. v. von W. Hubatsch (et al.), (Wissenschaftliche Abhandlungen der Arbeitsgemeinschaft für Forschung des Landes Nordrhein-Westfalen 30, 1964), S. 1.

${ }^{3}$ D.H. Arnold, Ausführliche und mit Urkunden versehene Historie der Königsbergischen Universität, 1 (1746), S. 60.

4 J. Serczyk, Albertyna Uniwersytet w Królewcu (1544-1945) [Albertina-Universität in Königsberg (1544-1945)], (1994), S. 22.

5 Serczyk, Albertyna, S. 25 
war die Blütezeit der Königsberger Hochschule, an der Polen, Litauer, Ankömmlinge aus Schlesien, Pommern, Livland, Bewohner des Kurlands und später aus Russland studiert haben. In allen oberen Fakultäten wurden seit 1640 Doktorwürden verliehen, wodurch der Einsatz besser qualifizierter Professorenkräfte gesichert wurde. Zuvor war es manchmal vorgekommen, dass auch eine Person ohne Magistertitel einen Professorenlehrstuhl übernommen hatte. Indessen waren die kommenden Jahre, u.a. unter der Herrschaft von Friedrich Wilhelm I. (1713-1740), nicht besonders günstig für die Entwicklung der Universität. Der König hatte keinerlei Interesse, den Universitätsrang durch intensive Entwicklung der Wissenschaft zu erhöhen. Die Universitätsabsolventen betrachtete er vor allem als Kandidaten für die Armee ${ }^{6}$. Als Ergebnis dieser Betrachtung können wir das Sinken der Beliebtheit der Hochschule am Ende des 18. und am Anfang des 19. Jahrhunderts beobachten. Die Albertina hatte den großen Teil ihrer Bedeutung verloren, insbesondere nach dem Tod von Immanuel Kant im Jahre 1804. Kant war seit 1755 als Privatdozent an der Philosophischen Fakultät beschäftigt, und sein Name hat viele junge Menschen nach Königsberg gezogen, die neue philosophische Richtungen kennenlernen wollten ${ }^{7}$. Nach seinem Tod wurde die Universität immer öfter als Provinzhochschule betrachtet, v.a. in Bezug auf das seit Jahrhunderten nicht geänderte Lehrsystem. Die Albertus-Universität wurde zu einer Akademie, die mit der Konkurrenz seitens der deutschen Hochschulen nicht Schritt halten konnte. Dorthin ist in dieser Zeit die wohlhabendere Jugend gereist. Durchschnittlich wurden in den Jahren 1802-1804/1805 an der Universität in Königsberg nur 132 Stu-

6 Ebenda, S. $58 \mathrm{f}$.

7 S. Dietzsch, Immanuel Kant. Eine Biographie, (2003), S. 65 f. 
denten aufgenommen ${ }^{8}$. Diese Tatsache hat u.a. dazu beigetragen, dass die sowieso nicht besonders reiche Albertus-Universität gänzlich verarmte.

Seit 1807 wurden Umstrukturierungsarbeiten an der Universität durchgeführt. Alle Angestellten an der Universität unterlagen jetzt dem allgemeinen Gerichtswesen und die Disziplinarangelegenheiten blieben nach wie vor beim Universitätsgericht. Die Reform hat zum großen Teil die didaktische Vorgehensweise umfasst. Bei allen Fakultäten war es jetzt möglich, die Unterrichtseinheiten als Übungen durchzuführen. Eine solche Unterrichtsform war bis jetzt nur an der Medizinischen Fakultät bekannt gewesen. Die Professoren hatten den direkten Kontakt zu den Studenten u.a. beim Seminarunterricht. Die Vorherrschaft des Vortrags, als der einzig anerkannten Lehrform, wurde zu dieser Zeit aufgehoben. Am schnellsten wurden die Änderungen in der Medizinischen Fakultät sichtbar. Es wurden nach und nach errichtet: Universitätsklinikum im Jahre 1809, Chirurgieklinikum im Jahre 1816, und 1830 wurde die neue Anatomieanstalt gegründet $^{9}$. In der Theologischen- und Philosophischen Fakultät ist die Zahl der Seminare auf 9 gestiegen, es wurden neue Lehrstühle und Institute gegründet. Die Zahl der Studenten in den 30er und 40er Jahren des 19. Jahrhunderts (angegeben separat im Sommerund Wintersemester) schwankte von 426 und 452 bis 318 und 285, die letzten Angaben beziehen sich auf das Wintersemester $1847 / 1848$ und das Sommersemester $1847^{10}$. Zum Vergleich: im Jahre 1910 studierten an der Universität 1400 Personen, angestellt

8 Archiwum Państwowe w Olsztynie [Staatsarchiv in Olsztyn] (weiter: APO), 1646/845, S. 36-37, 69.

9 Prutz, Die Königliche, S. 167 f.

10 APO, 1646/314. 
waren dort 158 Dozenten und Hochschullehrer, es gab 18 Seminare, 35 Institute und mehrere Kliniken ${ }^{11}$.

Drei Jahre vor dem Ausbruch des Krieges wurden aus der Philosophischen Fakultät die Naturwissenschaften ausgesondert. Aus ihnen wurde eine separate Fakultät gegründet. Die nächste, sechste Fakultät, die Landwirtschaftliche Fakultät, wurde 1943 auf Grundlage des bisherigen Landwirtschaftlichen Instituts gebildet ${ }^{12}$.

Die Akademie beendete ihre Tätigkeit im August 1944, zum 400jährigen Jubiläum der Universität. Nach dem Luftangriff wurde die Universität geschlossen. Die Unterrichtseinheiten wurden offiziell zum 28. Januar 1945 aufgehoben ${ }^{13}$.

\section{DiE KANZLEI, DIE REGISTRATUR, DAS UNIVERSITÄTSARCHIV}

Seit dem Anfang der Universitätstätigkeit wurden die Kanzleiaufgaben von einem Sekretär ausgeführt. Für diese verantwortungsvolle Funktion musste die jeweilige Person sehr gut ausgebildet sein. Oft wurde die Sekretärfunktion von einem Absolventen der Königsberger Akademie übernommen, wie im Fall von Martin Pflüger aus Nürnberg, der 1556, zwei Jahre nach seiner Magisterprüfung, die Stelle des Sekretärs bekommen hatte. Es ist auch manchmal vorgekommen, dass der Sekretär des Senats zusätzlich öffentliche Funktionen ausgeübt hat. Erasmus Landenberg von

11 Geheimes Staatsarchiv Preußischer Kulturbesitz (weiter: GStA PK), Rep.76 Va Sekt.11 Tit.V No 1 Bd IX.

12 E. Lang, Die Landwirtschaftliche Fakultät an der Albertus-Universität Königsberg, „Jahrbuch der Albertus-Universität zu Königsberg“, 2 (1952), S. 267.

13 G. von Selle, Geschichte der Albertus-Universität zu Königsberg in Preussen, (1956), S. 362. 
Friedland in Preußen, der von 1631 bis 1658 die Kanzlei des Senats führte, war gleichzeitig Sekretär beim Stadtgericht. Oder Johann Wahrt, ein Königsberger, Sekretär in den Jahren 1682-1696, war früher, seit 1678, als Hofgerichtsadvokat tätig. Das Amt wurde meistens über längere Zeit hinweg durch eine Person bekleidet. Zum Beispiel waren Christoph Ungermann über 30 Jahre (1558-1588) und Michael Störmer 32 Jahre (1598-1630) Sekretäre. Dies war sicher von Vorteil für die Kanzlei und Registratur, unter der Voraussetzung, dass sie auch gewissenhafte Menschen waren ${ }^{14}$.

$\mathrm{Zu}$ den Aufgaben des Sekretärs gehörten u.a. der Versand und die Registrierung diverser Schreiben. Für die ankommenden Schreiben wurde von ihm ein genaues Verzeichnis geführt, und für sonstige Tätigkeiten hatte der Sekretär vier Bücher zur Verfügung: „Decernententabelle, Stempelcontrolle, Distributionsbuch und das Buch worin die Urtheilsgebühre notirt werden, welche ad den Aerarium fallen "15. Darüber hinaus hat er Protokolle von den Sitzungen des Senats geführt. Die Entscheidungen des Senats, die mit aktuellen Prozessangelegenheiten nicht verbunden waren, wurden vom Sekretär als lose Blätter ad acta gelegt und dann in ein separates Buch eingetragen, das in der Registratur aufgehoben wurde. Der Sekretär nahm auch an den sog. Gerichtstagen des Rektors teil und führte die Protokolle. Die Registratur wurde in seine Obhut gegeben. Neben den strikt sachlichen Aufgaben hatte er auch andere. Bei der Verleihung der Doktorwürde hat er beispielsweise den Eid der Kandidaten angenommen.

Die andere Person, die in der Kanzlei angestellt war, war der Schreiber, die Aushilfe des Sekretärs. Man nannte ihn ein[en] simplex Copist oder Scriba. Zu seinen Aufgaben gehörten das

\footnotetext{
14 Arnold, Ausführliche, 2 (1746), S. 75 f.

15 APO, 1646/806, S. 1.
} 
Abschreiben der Dokumente und die Ausfertigung der Reinschrift. Der Schreiber trug auch alle sog. Publica-Angelegenheiten in separate Bücher ein, d.h. solche, die Spenden, Privilegien, Schulden, Fonds, Stipendien betrafen. Er schrieb auch Reskripte ab, deren Kopien von ihm für entsprechende Abteilungen oder Personen ausgefertigt wurden. In der Kanzlei befanden sich also neben den Büchern, die von dem Sekretär geführt wurden, auch andere: „liber donationum, liber privilegiorum, liber stipendiorum, liber obligationum, liber rescriptorum“. Der Schreiber fertigte auch die Berichte an, die im Namen des Senats an die Vorgesetzten verschickt wurden. Anhand der zugestellten Informationen erarbeitete er auch finanzielle Zusammenstellungen der Stipendienkassen und schrieb alle Anordnungen des Rektors und des Senats ins Reine. Sie wurden dann in der Schule „auf dem schwarzen Brett" ausgehängt. Als Vergütung für seine Arbeit bekam der Schreiber ein kleines Stipendium. Diese Stelle wurde meistens an Jura- oder Theologiestudenten vergeben. Viele bewarben sich, es war eine gute Möglichkeit, zusätzliches Gehalt zu bekommen, was bei den niedrigen Studentengeldern nicht ohne Bedeutung war ${ }^{16}$. Im Jahre 1795 wurde die Funktion des Protokollführers, die bisher dem Sekretär zugeteilt war, von dem Schreiber übernommen ${ }^{17}$. Am Anfang des 19. Jahrhunderts spielte der Schreiber eine immer wichtigere Rolle. Seit 1842 werden als Schreiber statt schlecht bezahlter Studenten erfahrene Schreiber mit guter Berufsvorbereitung angestellt. Diese übernehmen die Aufgaben des Kanzlisten, Protokollführers, Registratur- und Kalkulatorassistenten ${ }^{18}$.

16 APO,1646/1564, S. 142,143.

17 APO, 1646/1564, S. 184.

18 APO, 1646/248. 
Die Zweipersonenbesatzung der Kanzlei hat sich bis zu den Anfängen des 20. Jahrhunderts erhalten. Laut der Vorschrift aus dem Jahr 1906 sollte im Sekretariat noch eine dritte Person angestellt werden, die sog. Büroaushilfe. Zu den Aufgaben dieser Person gehörten u.a. die Führung des Briefjournals, Kanzleiarbeiten und die Postexpedition für den akademischen Senat sowie die Führung der Protokolle bei den Gerichtsverhandlungen ${ }^{19}$. Jedoch noch im Jahre 1910 hat der Universitätssenat an das Kuratorium eine Bitte gerichtet, diese Stelle zu besetzen ${ }^{20}$. Die Kanzlei, die anfangs als Senatskanzlei bezeichnet wurde, erfüllte in den späteren Jahren de facto die Funktion der allgemeinen Kanzlei.

Wie sah also die Organisation der Registratur und des Archivs der Universität aus, bei dem bescheidenen Personal und der immer größer werdenden Zahl der laufenden Angelegenheiten, die in das Journal eingetragen werden sollten?

Der Zustand der Registratur war offensichtlich besorgniserregend, denn im Protokoll des Senats vom 13. Juli 1768 finden wir die kurze Feststellung, dass „die [Registratur] in ziemliche Unordnung ist“"21. Drei Jahre vorher, im Jahre 1765, hatte der Rektor L'Estocq eine Bitte an den Senat gerichtet. Dessen Mitglieder sollten ihre Meinung über die Verbesserung der Organisation der Universität äußern. Es wurde damals gleichzeitig auch die Bewertung der Tätigkeit des Sekretärs durchgeführt im Bereich seiner Registraturaufsicht. Professor Jester hat die Angelegenheit wie folgt quittiert:

19 E. Wollenberg, Handbuch der Königlichen Albertus-Universität zu Königsberg Pr., (1908), S. 288.

20 GStA PK, Rep. 76 Va, Sekt. 11, Tit. V, No 1, Bd IX.

21 APO, 1646/1564, S. 140. 
Die kleine Registratur hielt er in möglicher Ordnung, und von hin und wieder sich ein Fehler ereignet, kann solche ohne große Geräusche redressiret worden ${ }^{22}$.

Das Unordnungsproblem in der Registratur wurde nicht lange ohne Lösung gelassen. Im Jahre 1850, bei der Übergabe der Registraturakten an das Universitätsgericht, wurde festgestellt, dass einige von ihnen fehlten, obwohl sie im Repertorium der reponierten Akten nachgewiesen waren. Die Angelegenheit wurde schnell geklärt. Reponierte Akten des Universitätsgerichts wurden im selben Schrank aufgehoben, in dem auch alte Senatsakten gelagert wurden. Vor Jahren hat man dem Referendarius Ewers befohlen, die Akten zu ordnen und aufzulisten, und dieser hat, wahrscheinlich aus Versehen, die Gerichtsakten aus dem ersten Fach in die untergeordneten Senatsakten mit aufgenommen. Man kann also annehmen, dass am Anfang des 19. Jahrhunderts der ganze Bestand der reponierten Registratur geordnet wurde. Vielleicht geschah dies auch, als 1862 die alten Universitätsgebäude in der Nähe des Doms im Kneiphof verlassen wurden und die Universität in die neuen Räumlichkeiten umzog.

Das erhaltene Verzeichnis der reponierten Akten aus der ersten Hälfte des 19. Jahrhunderts beinhaltet 266 Positionen und umfasst die Akten des Senats, der vier Fakultäten und des Universitätsgerichts. Es ist auch vorgekommen, dass unter einer Nummer mehrere (manchmal auch ein paar Dutzend) Volumina von ähnlichen Akten eingetragen wurden, u.a. 4 Volumina akademischer Protokolle 1711-1728, 55 Volumina alter verjährter und sonstiger Schuldklagen aus den Jahren 1740-1790 oder 13 alte Bücher aus den Jahren 1600-1700. Auf den letzten Seiten des Verzeichnisses wurden auch lose Akten ohne Nummern aus

22 APO, 1646/236, S. 329. 
den Jahren 1830-1839 genannt, die in der Registratur drei Fächer belegen. $\mathrm{Zu}$ den am ehesten erwähnten Akten in dieser Auflistung gehören alte Reskripte 1545, Belege und Quittungen 1600-1700, 10 Pakete alter Belege 1600-1706, 47 Volumina alter Testamentssachen 1650-1805 und 20 Volumina alter Stipendienakten von 1694. Erwähnt wurden auch die Satzung der Jurafakultät aus dem 17. Jahrhundert, Protokolle des Rektors aus dem 17. und 18. Jahrhundert, alte Edikte und Verordnungen aus dem Jahre 1747 sowie alte Matrikel aus dem Jahre 1804. Und in dem Verzeichnis überwiegen Streitangelegenheiten (Klagen, Schuldklagen) sowie Bagatellsachen in 271 Volumina aus den Jahren 1789-181023.

In den Akten für die reponierte Registratur wurde eigentlich die Bezeichnung Archiv nicht erwähnt. Dieser Begriff bezog sich anfänglich nur auf eine Kiste oder einen Schrank, die/der in dem Sitzungsraum des Senats (in Senatorio) stand. Die Eintragungen dazu finden wir im Buch der Juristischen Fakultät: „Curae Decani commisum est Scrinium, Archivum dictum, quod in Senatorio locum habet “24 und in den Akten der Theologischen Fakultät, wo wir Folgendes lesen können: „in Scrinio asservantur Seqventia Scripta “25. Man muss jedoch wissen, dass Scrinium in Senatorio ein allgemeines Archiv war, in das die Dekane nur die ältesten Bücher und Akten ihrer Fakultäten abgaben.

In seiner Monographie beschreibt Arnold sehr ausführlich die Lage der Stuben im alten Collegium:

Zur rechten Hand ist zur Seite der Juristische Hörsaal, und dem großen Auditoris gegenüber der Vorsaal der Stube, in welcher der Akademische Senat sowohl, als auch die Fakultäten ihre Seßiones

\footnotetext{
23 APO, 1646/153.

24 APO, 1646/229, S. 2-4.

25 APO, 1646/483, S. 1, 30.
} 
halten, und nach welcher das Gewölbe folget, darin die Registratur sich findet ${ }^{26}$.

Er erwähnt aber an keiner Stelle das Archiv.

Wir finden nicht viele Informationen über den frühesten Bestand des Universitätsarchivs, d.h. über Dokumente und lose Akten. Aus einer unscheinbaren Notiz aus dem Jahre 1679, die vom Sekretär Erasmus Landenberg dem Jüngeren angefertigt worden war, erfahren wir, dass im Archiv zu dieser Zeit ein Privileg der Universität vom 18. April 1557 sowie seine Bestätigung (Confirmatio) und ein Diplom auf Pergament mit einem Siegel in einer silbernen Kapsel aufgehoben wurden. Dieses Diplom wurde durch den polnischen König Zygmunt August am 28. März 1560 verliehen ${ }^{27}$. Ein Teil der Universitätsbibliothek bestand aus einer Dokumentensammlung aus den Jahren 1544-1619, die dort unverändert gelagert wurde ${ }^{28}$.

Auf einigen nicht datierten, zusammengehefteten Blättern, die im Staatsarchiv in Königsberg mit dem Titel Verzeichnisse des 17. Jahrhunderts betreffend datierte und undatierte Akten sowie Rechnungen des 16. u. 17. Jahrhunderts versehen waren, wurden auch lose Akten aufgenommen: Reskripte, Rechnungen, Anträge und Gesuche $^{29}$.

26 Arnold, Ausführliche, 2, S. 43.

27 APO, 1646/236, S. 47.

28 Arnold, Ausführliche, 2, S. 45.

29 APO, 1646/2. 


\section{DER ARCHIVBESTAND „ALBERTUS-UNIVERSITÄT IN KÖNIGSBERG"}

Die Akten der Universität, die heute im Staatsarchiv in Olsztyn aufgehoben werden, bilden einen separaten Bestand, der aus 1892 Archiveinheiten besteht. Das bedeutet etwa 38 laufende Meter Akten aus den Jahren 1554-1933. Jedoch stammen die erwähnten Archivalien nicht direkt aus der Universitätsregistratur, sondern sind ein Teil eines Depots, das von den Universitätsmitarbeitern in den Jahren 1919 und $1921 \mathrm{im}$ Staatsarchiv in Königsberg eingerichtet wurde. Das Findbuch für das Depot, manchmal auch Repertorium genannt, wurde in Königsberg durch die Archivare Schultz und Max Hein, den späteren Direktor des Königsberger Staatsarchivs, angefertigt. Es beinhaltet 2750 Archiveinheiten aus den Jahren $1544-1935^{30}$. Das Findbuch besteht aus zwei Teilen. Der erste Teil beinhaltet 28 Serien, der andere 26. Die Akten wurden geordnet, ohne dass man sich an den ursprünglichen Plan der Universitätsregistratur gehalten hat. Es ist leicht bemerkbar, dass sich in Olsztyn etwa 68\% des Königsberger Depots befinden, die Verluste umfassen über 850 Akten. Darüber hinaus finden wir im Staatsarchiv in Olsztyn keine früher im Text erwähnten Privilegien, Diplome, Matrikel oder Satzungsoriginale, weil diese aus logischen Gründen nicht im Depot, sondern im Universitätsarchiv hinterlassen wurden.

Man muss auch das Anfangsdatum erklären, das für den Bestand angenommen wurde, nämlich das Jahr 1554, in dem die Satzung der Universität unterschrieben wurde. Obwohl sich in dem Bestand das Volumen befindet, dessen chronologischer

30 B. Wacławik, Archiwalia do dziejów Uniwersytetu Królewieckiego przechowywane w Archiwum Państwowym w Olsztynie [Archivalien zur Geschichte der Königsberger Universität in dem Staatsarchiv in Olsztyn], „Archeion“, 95 (1995), S. 60. 
Umfang noch in der Universitätsregistratur für die Zeit 1544-1810 festgelegt wurde, ist die beigefügte Verordnung des Herzogs Albrecht aus dem Jahre 1544 eine de facto später angefertigte Abschrift ${ }^{31}$.

Eine der ältesten Archivalien in dem Bestand sind die Satzungen und Privilegien, wobei von den in dem Königsberger Repertorium erwähnten 12 in Olsztyn nur 5 aufgehoben werden, nämlich die Satzung aus dem Jahre 1554 in der Abschrift, die Satzung der Medizinischen Fakultät aus dem Jahre 1618 und die der Philosophischen Fakultät aus dem Jahre 1561, Privilegien und Reglements aus dem 18. Jahrhundert sowie die Satzung aus dem Jahre 1843. Die erhaltenen Protokolle des Rektors beziehen sich auch auf die frühesten Jahre der Universitätstätigkeit. Trotz der Lücken bilden sie eine ziemlich geschlossene Sammlung von zusammengehefteten und losen Akten aus den Jahren 1642-1689, 1725, 1741-1752. Ihre Ergänzung sind die Protokolle des Senats aus den Jahren 1638, 1682-1694, 1724-1732, 1756-1802, 1897-1904, leider sind auch diese nicht komplett.

Die Akten der vier Fakultäten haben sich quantitativ und chronologisch in unterschiedlichem Umfang erhalten. Es werden in Olsztyn zurzeit nur 19 Akten von den 40 in Königsberg inventarisierten Theologiefakultätsakten aufbewahrt. Dies sind die Senatsakten betreffend die Fakultät aus den Jahren 1621-1835, Protokolle von den Fakultätssitzungen 1749-1760, Akten über die Anstellung von Professoren 1609-1750, Vorlesungsverzeichnisse 1773-1809, Akten des litauischen und polnischen Seminars 1718-1729, Akten des theologischen und homiletischen Seminars $1821-1852$.

31 APO, 1646/236. Zwei Schreiben 1545 und 1546 (Original?) kann man noch in den Akten APO1646/1591, 1646/841 finden. 
Wesentlich weniger Akten haben wir von der Juristischen Fakultät, nur 8 von 24 Akteneinheiten, unter anderem zwei Fakultätsbücher (Liber responsorum) 1721-1733 und 1750-1764, Akten über die Anstellung der Professoren 1610-1698, Akten über die abgelegten Prüfungen sowie über das Studium der Kameralwissenschaften 1825-1835.

Die Medizinische Fakultät ist im Bestand vertreten mit den Fakultätsbüchern (Liber consiliorum et responsorum), 5 Bänden aus den Jahren 1716-1785, einem Protokollbuch der Fakultät 1623-1715, weiter den Acta Generalia 1641-1741 sowie über 90 Volumina der Fakultätsakten aus den Jahren 1751-1879.

60 Akten, insgesamt gab es in Königsberg 105, aus den Jahren 1561 - 1917 betreffen die Philosophische Fakultät: 3 Fakultätsbücher aus den Jahren 1731-1845, darüber hinaus Fakultätsakten, darunter die ältesten aus dem Jahre 1815 (nicht komplett) und aus den Jahren 1880-1900. Letztere bilden eine lückenlose Einheit. Weiterhin gibt es 11 Volumina über die Verleihung der Doktorwürde in den Jahren 1879-189732.

Zahlreiche Serien bestehen auch aus Personalakten der Universitätsmitarbeiter: 136 Akteneinheiten, Stipendienakten 1600-1917; 83 Akteneinheiten (AE), Rechnungen und Anhänge dazu1663-1820; 51 AE, Testamente 1620-1832; 172 AE und Darlehen 1632-1912; 175 AE.

Zuletzt sollte man noch die Akten erwähnen, die ein Teil des Bestandes Albertus-Universität zu Königsberg sind, aber im Königsberger Repertorium sich nicht befinden. Es sind die Akten des Landwirtschaftlichen Instituts der Albertus-Universität aus den Jahren 1888-1926, die zum größten Teil Arbeiten über Land-

32 Für die Doktoranden an der Philosophischen Fakultät wurde eine separate Serie im Königsberger Repertorium eingerichtet: Disputationen und Promotionen. 
wirtschaft, Tierzucht und Botanik beinhalten. Eine Akte betrifft aber auch die Organisation des Instituts und eine andere die Tätigkeit seiner Bibliothek. Zusammen bilden sie eine Serie mit 22 Akteneinheiten. Darüber hinaus sind auch eigene Akten des Studentenkorps Littuania aus den Jahren 1836-1933 erhalten geblieben, die jedoch in dem Bestand gelassen wurden. 


\section{ARCHIWUM UNIWERSYTETU ALBRECHTA W KRÓLEWCU PRZECHOWYWANE W ZASOBIE ARCHIWUM PAŃSTWOWEGO W OLSZTYNIE}

(STRESZCZENIE)

Uniwersytet w Królewcu, założony przez Albrechta Hohenzollerna w 1544 r., powstał przede wszystkim z konieczności kształcenia urzędników, nauczycieli, teologów dla potrzeb organizacji świeckiego państwa oraz kształtowania nowej tożsamości religijnej i społecznej poddanych księcia. Nauką wiodącą w Królewcu była teologia, a pozostałe nauki wykładane były zgodnie z doktryną luterańską. Studenci mieli do wyboru cztery wydziały: Teologiczny, Medyczny i Prawny zwane wyższymi oraz tzw. niższy Filozoficzny. Dopiero w latach 30. XX w. nauki przyrodnicze wydzielono z Wydziału Filozoficznego, w 1943 r. powstał Wydział Rolny. Od 1640 r. na wszystkich wyższych wydziałach odbywały się promocje doktorskie. Lata rozkwitu uczelni przypadają na XVII w., kiedy to w Królewcu studiują Polacy, Litwini, przybysze ze Śląska, Pomorza, Inflant, Kurlandii, a później i Rosji. Kolejne lata nie były korzystne dla rozwoju Uniwersytetu. Dopiero z początkiem XIX w. przeprowadzono reformę uczelni, również w zakresie dydaktyki. Uczelnia zakończyła swoją działalność w połowie sierpnia 1944 r. Zajęcia oficjalnie zostały zawieszone 28 stycznia $1945 \mathrm{r}$.

Od początków uniwersytetu czynności kancelaryjne wykonywał sekretarz, który z racji sprawowanej funkcji musiał być osobą dobrze wykształconą. Drugą osoba zatrudnioną w kancelarii był pisarz, określany jako kopista bądź skryba. Jego ranga rośnie w XIX w., kiedy na tym stanowisku zaczęto zatrudniać doświadczonych pisarzy. Kancelaria, którą najpierw nazywano kancelarią senatu, później de facto pełniła rolę kancelarii ogólnej. Przy skromnym personelu (do początku XX w. zatrudniała dwie osoby) organizacja registratury nie zawsze była wzorowa. W pierwszej połowie XIX w. uporządkowano zasób registratury repo- 
nowanej. Zachowany z tego okresu spis zawiera 266 pozycji i obejmuje akta senatu, czterech fakultetów oraz sądu uniwersyteckiego począwszy od roku 1545. Właściwie w aktach dla registratury reponowanej nie stosowano określenia archiwum. Ten termin przypisany był początkowo tylko skrzyni bądź szafie, która znajdowała się w sali posiedzeń senatu. Niewiele informacji znajdujemy o najwcześniejszym zasobie archiwum uniwersyteckiego.

Akta uniwersytetu (z lat 1554-1933), przechowywane obecnie w Archiwum Państwowym w Olsztynie, tworzą odrębny zespół liczący 1892 j.a. (ponad $39 \mathrm{mb}$ ). Nie pochodzą one jednak bezpośrednio z registratury uniwersyteckiej, lecz są częścią depozytu, który uczelnia złożyła w latach 1919 i 1921 w Archiwum Państwowym w Królewcu. Repertorium dla depozytu sporządzone w Królewcu wykazuje 2750 j.a. z lat 1544-1935. W Olsztynie znajduje się około 68\% depozytu królewieckiego, a straty obejmują ponad 850 j.a. W tym zespole do starszych archiwaliów zaliczyć trzeba statuty i przywileje, m.in. statut uczelni z 1554 r. czy Wydziału Filozoficznego z 1561 r. Protokoły rektora mimo luk odnoszą się do wczesnych lat działalności uczelni (1642-1752). Ich uzupełnieniem są protokoły senatu z lat 1638-1904, również niekompletne. W różnym zakresie ilościowym, jak i chronologicznym zachowały się akta czterech Wydziałów.

Opracowane przez Autorkę/Bearbeitet von der Verfasserin 